\title{
Clarifications to Questions and Criticisms on the Johansen-Ledoit-Sornette Financial Bubble Model
}

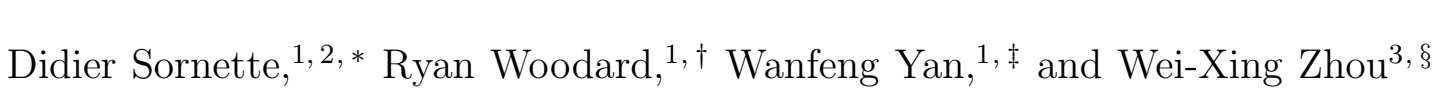 \\ ${ }^{1}$ Department of Management, Technology and Economics, \\ ETH Zurich, Kreuzplatz 5, CH-8032 Zurich, Switzerland \\ ${ }^{2}$ Swiss Finance Institute, c/o University of Geneva, \\ 40 blvd. Du Pont d'Arve, CH-1211 Geneva 4, Switzerland \\ ${ }^{3}$ School of Business, East China University of Science and Technology, Shanghai 200237, China \\ The Johansen-Ledoit-Sornette (JLS) model of rational expectation bubbles with \\ finite-time singular crash hazard rates has been developed to describe the dynamics \\ of financial bubbles and crashes. It has been applied successfully to a large variety \\ of financial bubbles in many different markets. Having been developed over a decade \\ ago, the JLS model has been studied, analyzed, used and criticized by several re- \\ searchers. Much of this discussion is helpful for advancing the research. However, \\ several serious misconceptions seem to be present within this literature both on theo- \\ retical and empirical aspects. Several of these problems stem from the fast evolution \\ of the literature on the JLS model and related works. In the hope of removing pos- \\ sible misunderstanding and of catalyzing useful future developments, we summarize \\ these common questions and criticisms concerning the JLS model and synthesize the \\ current state of the art and existing best practice.
}

Keywords: JLS model, financial bubbles, crashes, log-periodic power law, fit method, sloppiness, taboo search, bootstrap, probabilistic forecast.

*Electronic address: dsornette@ethz.ch

${ }^{\dagger}$ Electronic address: rwoodard@ethz.ch

‡Electronic address: wyan@ethz.ch

$\S$ Electronic address: wxzhou@ecust.edu.cn 


\section{INTRODUCTION}

The Johansen-Ledoit-Sornette (JLS) model [1 4] has been developed to describe the dynamics of financial bubbles and crashes. The model states that bubbles are not characterized by an exponential increase of price but rather by a faster-than-exponential growth of price. This phenomenon is generated by behaviors of investors and traders that create positive feedback in the valuation of assets leading to unsustainable growth ending with a finite-time singularity at some future time $t_{c}$.

One can identify two broad classes of positive feedback mechanisms. The first technical class includes (i) option hedging [5], (ii) insurance portfolio strategies (see paragraph 1 on page 380 of Ref. [6] stating that "Positive feedback trading is also exhibited by buyers of portfolio insurance...") and Refs. [7, 8]), (iii) market makers bid-ask spread in response to past volatility [9, 10], (iv) learning of business networks and human capital build-up [11, 12], (v) procyclical financing of firms by banks (boom vs contracting times) [13], (vi) trend following investment strategies, (vii) asymmetric information on hedging strategies 14] (viii) the interplay of mark-to-market accounting and regulatory capital requirements [15, 16]. The second class of positive feedback mechanisms is behavioral and emphasizes that positive feedbacks emerge as a result of the propensity of humans to imitate, of their social gregariousness and the resulting herding. This critical time $t_{c}$ of the model is interpreted as the end of the bubble, which is often but not necessarily the time when a crash occurs in the actual system. During this growth phase, the tension and competition between the value investors and the noise traders create deviations around the hyperbolic power law growth in the form of oscillations that are periodic in the logarithm of the time to $t_{c}$. Combining these two effects, this model succinctly describes the price during a bubble phase as a log-periodic (hyperbolic) power law (LPPL).

Since its introduction, the JLS model has been used widely to detect bubbles and crashes ex-ante (i.e., with advanced documented notice in real time) in various kinds of markets such as the 2006-2008 oil bubble [17], the Chinese index bubble in 2009 [18], the real estate market in Las Vegas [19], the U.K. and U.S. real estate bubbles [20 24], the Nikkei index anti-bubble in 1990-1998 [25] and the S\&P 500 index anti-bubble in 2000-2003 [26]. Other recent studies performed in an ex-post mode include the Dow Jones Industrial Average historical bubbles [27], the corporate bond spreads [28], a Polish stock market bubble [29], 
some western stock market bubbles [30], U.K. stock market bubbles [31], the Brazilian real (R\$) - US dollar (USD) exchange rate [32], 2000-2010 world major stock indices [33], South African stock market bubbles [34], the US repurchase agreements market [35] and emerging markets [36, 37]. Moreover, new experiments in ex-ante bubble detection and forecasts have been launched since November 2009 in the Financial Crisis Observatory at ETH Zurich [3840]. In many cases, market risk is contagious [41] and market corrections and crashes occur successively in a short period [42] including the latest financial crisis, which is partially due to globalization and assets diversification such that the US stock market and world financial stock market exhibit long-range cross-correlations [43, 44].

Having been developed over a decade ago, the JLS model has been studied, used and criticized by many researchers including Feigenbaum [45], Chang and Feigenbaum [46, 47], van Bothmer and Meister [48], Fry [22], and Fantazzini and Geraskin [49]. The most recent papers addressing the pros and cons of past works on the JLS model are written by Bree and his collaborators [50, 51]. Many ideas in these last two papers are correct, pointing out some of the inconsistencies in earlier works. However, there are some serious misunderstandings concerning both the theoretical and empirical parts of the model. Therefore, it is necessary to address and clarify the misconceptions that some researchers seem to hold concerning the JLS model and to provide an updated, concise reference on the JLS model.

The structure of this paper is as follows. Section [1] discusses the questions about the theory and derivation of the JLS model. The questions on fitting methods of the model are commented on Section III, Issues on probabilistic forecast will be addressed in Section IV. We conclude in Section $\mathrm{V}$.

\section{PRESENTATION AND REMARKS ON THE THEORETICAL FOUNDATION OF THE JLS MODEL}

We will give the derivation of the JLS model first in this section. Then we discuss three issues related to the derivation and the proper parameter ranges. 


\section{A. Derivation of the JLS model}

The JLS model starts from the rational expectation settings of [52], where the observed price $p_{o}$ of a stock can be written as

$$
p_{o}=p^{*}+p
$$

where $p^{*}$ and $p$ represent respectively the fundamental value and the bubble component. Eq. (11) shows that the price is a linear combination of the fundamental value and the bubble component. The JLS model specifies the dynamics of the bubble component independently of the dynamics of the fundamental price. The later can be specified according to standard valuation models, for instance leading to the usual geometrical random walk benchmark. The JLS model adds to this featureless fundamental price the so-called log-periodic power law structure, which is used to diagnose the presence of bubbles. Lin et al. [53] have considered a self-consistent mean-reverting process for $p^{*}$ that makes consistent the calibration of the observed price $p_{o}$ by the JLS model.

The JLS model starts from the assumption that the dynamics of the bubble component of the price satisfies a simple stochastic differential equation with drift and jump:

$$
\frac{d p}{p}=\mu(t) d t+\sigma d W-\kappa d j
$$

where $p$ is the stock market bubble price, $\mu(t)$ is the drift (or trend) and $d W$ is the increment of a Wiener process (with zero mean and unit variance). The term $d j$ represents a discontinuous jump such that $j=0$ before the crash and $j=1$ after the crash occurs. The loss amplitude associated with the occurrence of a crash is determined by the parameter $\kappa$. Each successive crash corresponds to a jump of $j$ by one unit. The dynamics of the jumps is governed by a crash hazard rate $h(t)$. Since $h(t) d t$ is the probability that the crash occurs between $t$ and $t+d t$ conditional on the fact that it has not yet happened, we have $E_{t}[d j]=1 \times h(t) d t+0 \times(1-h(t) d t)$ and therefore the expectation of $d j$ is given by

$$
E_{t}[d j]=h(t) d t
$$

Under the assumption of the JLS model, noise traders exhibit collective herding behaviors that may destabilize the market. The model assumes that the aggregate effect of noise traders can be accounted for by the following dynamics of the crash hazard rate:

$$
h(t)=B^{\prime}\left(t_{c}-t\right)^{m-1}+C^{\prime}\left(t_{c}-t\right)^{m-1} \cos \left(\omega \ln \left(t_{c}-t\right)-\phi^{\prime}\right) .
$$


The cosine part of the second term in the r.h.s. of (4) takes into account the existence of possible hierarchical cascades [54] of accelerating panic punctuating the growth of the bubble, resulting from a preexisting hierarchy in noise trader sizes [55] and/or the interplay between market price impact inertia and nonlinear fundamental value investing [56]. Expression (4) also contains a hyperbolic power law growth ending at a finite-time singularity, which embodies the positive feedbacks resulting from the technical and behavioral mechanisms summarized above in the Introduction.

The non-arbitrage condition expresses that the unconditional expectation $E_{t}[d p]$ of the price increment must be 0 , which leads to

$$
\mu(t) \equiv \mathrm{E}\left[\frac{d p / d t}{p}\right]_{\text {no crash }}=\kappa h(t)
$$

by taking the expectation of (22). Note that $\mu(t) d t$ is the return $\frac{d p}{p}$ over the infinitesimal time interval $d t$ in the absence of crash. Using this and substituting (44) and integrating yields the so-called log-periodic power law (LPPL) equation:

$$
\ln E[p(t)]=A+B\left(t_{c}-t\right)^{m}+C\left(t_{c}-t\right)^{m} \cos \left(\omega \ln \left(t_{c}-t\right)-\phi\right)
$$

where $B=-\kappa B^{\prime} / m$ and $C=-\kappa C^{\prime} / \sqrt{m^{2}+\omega^{2}}$. Note that this expression (4) describes the average price dynamics only up to the end of the bubble. The JLS model does not specify what happens beyond $t_{c}$. This critical time $t_{c}$ is the termination of the bubble regime and the transition time to another regime. The parameter $t_{c}$ represents the non-random time of the termination of the bubble. However, its precise value is not known with absolute precision, and its estimation can be written as

$$
t_{c}^{\text {estimated }}=t_{c}^{\text {true }}+\epsilon,
$$

where $\epsilon$ is an error term distributed according to some distribution, while $t_{c}^{\text {true }}$ is deterministic. Lin and Sornette [57] have recently extended the modeling to include a stochastic mean reversal dynamics of the critical time $t_{c}$, thus capturing the uncertain anticipation of investors concerning the end of the bubble.

\section{B. Why $m$ should be between 0 and 1? A balancing act}

The mechanism of positive feedback leading to faster-than-exponential price growth is captured by the exponent $m$, which should fall in the range $[0,1]$. A negative value of $m$ 
would correspond to unrealistic diverging prices in finite time. A value of $m$ larger than 1 would correspond to a decelerating price.

1. For $m<1$, the crash hazard rate accelerates up to $t_{c}$ but its integral up to $t$, which controls the total probability for a crash to occur up to $t$, remains finite and less than 1 for all times $t \leq t_{c}$. It is this property that makes it rational for investors to remain invested, knowing that a bubble is developing and that a crash is looming. Indeed, there is still a finite probability that no crash will occur during the lifetime of the bubble and beyond, so there is a chance for investors to gain from the ramp-up of the bubble and walk away unscathed. The excess return $\mu(t)=\kappa h(t)$ is the remuneration that investors require to remain invested in the bubbly asset, which is exposed to a crash risk. The crash hazard may diverge as $t$ approaches a critical time $t_{c}$, corresponding to the end of the bubble.

2. Within the JLS framework, a bubble exists when the crash hazard rate accelerates with time. According to (4), this imposes $m<1$ and $B^{\prime}>0$. That is, $m \geq 1$ cannot lead to an accelerating hazard rate.

3. Finally, the condition that the price remains finite at all time, including $t_{c}$, imposes that $m>0$.

Bree \& Joseph [50] note astutely that imposing the constraint $m<1$ might be detrimental to the discovery of the best fit of the LPPL model to a given price time series. Specifically, they write: "We presume that the reason that any fit with was rejected is because then the increase in the index is exponentially declining whereas the underlying mechanism requires it to be increasing. An alternative technique would have been to place no restriction on the value of $m$, and if a value of $m>1$ is found, to reject the model, as we have done for the requirement that the fitted LPPL never decreases."

In other words, Bree \& Joseph [50] propose to relax the condition $0<m<1$ because the existence of best fits that have exponents outside this range can be used as a diagnostic that no JLS-type bubble is present. There is merit in this reasoning but one must also take into account the large noise amplitude of the empirical price time series together with the nonlinear nature of the LPPL model, which make not fully reliable the selection of best fits solely based on the minimization of the residual root-mean-square errors (RMSE). We suggest that the use of constraints is to impose that the statistical calibration exercises pass the financial conditions of good sense (or "smell test", to paraphrase the term used by Robert Solow in his testimony to the US House Committee on Science and Technology, 
Subcommittee on Investigations and Oversight in July 2010 in the aftermath of the financial crisis). Other methods can be developed to address the compromise between best fit and meaningfulness, by addressing directly the problem of noise using bootstrap and ensemble methods, some of which having been already implemented in the advance tests of the ETH Zurich financial crisis observatory [38-40].

Finally, this condition $0<m<1$ should not be understood as "protecting" the JLS model, i.e., forcing the formula to fit the data. Indeed, one should not confuse the fact of restricting the range of $m$ values in the fitting procedure with the process of qualifying or not the existence of a bubble. In the many studies mentioned above, other selection procedures have been used than based on the value of the exponent $m$.

\section{Non-negative risk condition}

van Bothmer and Meister derived a constraint on the variables of the JLS model [48] from the statement that the crash rate should be non-negative. It states that:

$$
b:=-B m-|C| \sqrt{m^{2}+\omega^{2}} \geq 0 .
$$

Most current research using the JLS model has taken this restriction into consideration. It is among the basic restrictive filters for identifying bubbles in a more modern framework. In [58 61], the parameter $b$ in (8) is even used as a key trait implemented within a pattern recognition method to detect market rebounds.

\section{Decreasing price and decreasing expected price in the JLS model}

The condition derived by Graf v. Bothmer and Meister [48] is equivalent to the statement that the expected price variation in the absence of crash is non-negative, as seen from expression (5). This equivalence is emphasized by Bree and Joseph [50], who propose to use this requirement as a testable prediction. And they note that many of the case studies reported in Johansen and Sornette [36] and in Sornette and Johansen [62] have their LPPL fits that exhibit a negative slope some of the time. Since the LPPL fit is assumed to capture the expected price dynamics, a negative slope of the price at certain times is in contradiction with expression (5), given that the crash hazard rate $h(t)$ is non-negative. Bree and Joseph 
[50] then conclude that this fact is sufficient to reject the martingale condition as being the mechanism underlying the LPPL to pre-crash bubbles, at least for a large fraction of them. Their point is well taken and convincing. However, another viewpoint is that condition (8) associated with the non-negativity of $h(t)$ and $\mu(t)$ via the martingale condition offers a classification of bubbles in different types, those that obey the martingale condition and those that do not. Indeed, we do not believe that all bubbles are the same. For instance, we have reported evidence in the past that some bubbles are "fearful" while others are "fearless" [63]. Moreover, one can imagine other ways to cure the possible occurrence of negative values of the parameter $b$ given by expression (8), which considers hierarchies of crashes, without the need to abandon the martingale condition.

We stress that this should not be confused with a more naive view that, during a bubble following the JLS process, price has to always be increasing. Indeed, the definition of the JLS model includes implicitly the stochastic term $\sigma d W$ as in expression (2). In expectations, this term disappears as does any unbiased random walk whose average position remains identically zero while exhibiting realizations with significant deviations, hence it is not included in the description of the initial JLS paper. In the presence of this stochastic term $\sigma d W$, the price can exhibit transient negative spells, even if the expected trend should be positive according to expression (5). This formulation is nothing but that of the rational expectation of Blanchard and Watson [52], which follows exactly the same procedure, with a stochastic component which does not play a role in the specification of the crash hazard rate relationship to the $\mu$ term, but is present to ensure that the realized price can indeed decrease transiently, while the price should only increase in expectations.

\section{E. Faster-than-exponential growth in the JLS model}

One of the fundamental differences between the JLS model and standard models of financial bubbles is that the JLS model claims that the price follows a faster-than-exponential growth rate during the bubble. It is necessary to emphasize this statement as many researchers make mistakes here. For example, Bree and Joseph wrote "exponential growth is posited in the LPPL" in several places in [50].

Financial bubbles are generally defined as a transient upward acceleration of prices above the fundamental value [64 66]. However, identifying unambiguously the presence of a bubble 
remains an unsolved problem in standard econometric and financial economic approaches [67, 68], due to (i) the fact that the fundamental value is in general poorly constrained and (ii) the difficulty in distinguishing between an exponentially growing fundamental price and exponentially growing bubble price. As we have already described, the JLS model defines a bubble in terms of faster-than-exponential growth [69]. Thus, the main difference with standard bubble models is that the underlying price process is considered to be intrinsically transient due to positive feedback mechanisms that create an unsustainable regime. See for instance Ref.[18] where this is made as clear as possible.

\section{F. Which kind of bubbles can be detected by the JLS model?}

In page 4 of Ref.[50], three claims are outlined. One of them states that: "Financial crashes are preceded by bubbles with fluctuations. Both the bubble and the crash can be captured by the LPPL when specific bounds are imposed on the critical parameters $\beta$ and $\omega^{\prime \prime}$, where $\beta$ is presented as $m$ in this paper.

For further clarification, this above claim is not entirely correct because crashes can be endogenous or exogenous. The JLS model is suitable only for endogenous crashes! Or more precisely, the JLS model is for bubbles, not for crashes. Endogenous crashes are preceded by the bubbles that are generated by positive feedback mechanisms of which imitation and herding of the noise traders are probably the dominant ones among the many positive feedback mechanisms inherent to financial system. Johansen and Sornette [69] identify 49 outliers in the distribution of financial drawdowns, of which 25 can be classified as endogenous crashes preceded by speculative bubbles, 22 as attributable to exogenous events and fundamentally unpredictable and 2 as associated with the Japanese anti-bubble [25]. Restricting to the world market indices, they find 31 outliers, of which 19 are endogenous, 10 are exogenous and 2 are associated with the Japanese anti-bubble. Although the endogenous outliers are more frequent than the exogenous ones, the exogenous outliers still constitute a quite large portion. This should not be too surprising as financial markets are still impacted by news of all types that lead investors to reassess their expectations of future risk-adjusted returns and therefore to rebalance their portfolio allocations. In fact, according to the efficient market hypothesis, all crashes should be exogenous, i.e. driven by external unanticipated news. The surprising finding of this last decade, notably based on the JLS-LPPL model, has been to 
demonstrate that about two-thirds of crashes are of an endogenous nature, thus changing completely the paradigm of standard financial thinking. It is thus important to stress that, while relevant for about two-thirds of the observed crashes, the JLS model cannot capture all of the crashes in the market. Only endogenous crashes that are preceded by bubbles can be described by the JLS model. And when we write "describe", we mean that the probability of crashes can be estimated, but the specific unfolding of each crash and the post-crash dynamics is beyond the premise of the JLS model.

\section{FITTING PROBLEMS CONCERNING THE JLS MODEL}

\section{A. Extensions of the JLS model and their calibration}

The form of the JLS model represented by expression (6) is called the first-order LPPL Landau JLS model. Extensions have been proposed, essentially amounting to choose alternative forms of the crash hazard rate $h(t)$ that replace expression (4). Let us mention the so-called second-order and third-order LPPL Landau models [25, 26, 70 72], the Weierstrasstype LPPL model [73, 74], the JLS model extended with second-order and third-order harmonics [19, 34, 75, 76] and the JLS-factor model in which the LPPL bubble component is augmented by other financial risks factors [77, 78]. We should also mention that a nonparametric estimation of the log-periodic power law structure has been developed to complement the above parametric calibrations [79]. These extensions are warranted by the fact that the positive feedback mechanisms together with the presence of the symmetry of discrete scale invariance can be embodied in a general renormalization group equation [73], whose general solution is the generalized Weierstrass LPPL model. Then, the first-order LPPL Landau JLS model can be considered to be just the first term in a general log-periodic Fourier series expansion of the general solution. Therefore, further away from the critical time $t_{c}$, corrections from the first-order expression can be expected to be relevant, depending on the context. In addition, nonlinear extensions to the renormalization group are embodied partially in the second-order and third-order LPPL Landau models, which extend the time domain over which the model can be calibrated to the empirical data [54].

Sornette and Johansen [54] discussed the difference between the fitting results obtained using the first order and the second order LPPL Landau-type JLS models. They used daily 
prices of the S\&P 500 index from 1980 to 1987. The results show that the fitting result of the second order form is much better than the first order form, as based on the measure of residual sum of squares. A standard Wilks test of nested hypotheses confirms the fact that the second-order form provides a statistically significant improvement over the firstorder form (recall that the first-order LPPL Landau formula is recovered as a special case of the second-order LPPL Landau formula, hence the first model is nested within the second model).

We reproduce the fitting results from [54] in Fig. 11 to give an intuition on the difference between the first order and second order LPPL Landau fits. When fitting the first-order LPPL Landau formula over the time period from January 1980 to September 1987 (dashed thin line), we observe that the first-order LPPL Landau formula accounts reasonably well for the price dynamics from 1980 to 1985 but then becomes completely out of phase in the remaining two years before the crash. When fitting the first-order LPPL Landau formula in the interval from July 1985 to the end of September 1987 (continuous thin line) as done initially in Ref.[54], we observe that the first-order LPPL Landau formula accounts reasonably well for the data from mid-1985 to the peak in October 1987 but is completely out of phase with the price in the earlier time period from 1980 to 1985. In contrast, the second-order LPPL Landau formula (continuous thick line) provides a good fit over the whole period from January 1980 to September 1987. These results help explain why the results quoted by Bree et al. [51] for time windows of 834 trading days may be questionable.

Notwithstanding the improvement provided by the second-order LPPL Landau model for large time windows, it is sufficient in many cases to use the first-order version just to get a diagnostic of the presence of a bubble. This is true even when the time window is larger than 2-3 years. For instance, the first-order LPPL Landau model was implemented within a pattern recognition method [58, 59, 61] with time windows of up to 1500 days. The key to the reported performance in forecasting is the combination of bubble diagnostics at multiple time scales, with common model parameters associated with robustness [58, 59, 61].

\section{B. Selection of the start of the time window}

A common question arising in fitting the JLS model is to decide which date $t_{1}$ should be selected as the beginning of the fitting time window. Bree and Joseph [50] use a more 
consistent approach in defining the start time of the bubbles they analyze than the papers published from 1998 to 2000 that they compare with. It is worthwhile to mention that there are other procedures implemented systematically in the more recent Refs. [18, 38, 49, 59, 60, 80], in which multiple starting dates $t_{1}$ 's are selected to make the prediction more statistically robust. The findings of these articles is that focusing on a single $t_{1}$ corresponding to a single time window in which to perform the fit - may be unreliable and an ensemble of fits with different $t_{1}$ is recommended. Indeed, this reflects the fact that the start of a bubble is an elusive concept, as the early deviation of the observed price from its fundamental value is small in the first month and even years of the bubble, so the definition of the start date is somewhat fuzzy and degenerate.

\section{Should price or log-price be fitted?}

Sornette and Johansen [1] argue that log-price should be used when the amplitude of the expected crash is proportional to the price increase during the bubble. This prescription has been followed in many of the studies on the JLS model [18, 20, 38 40, 59, 61, 80]. This is because (6) is derived from (2), which assumes that the changing price $d p$ is proportional to the price $p$. Therefore, this statement is in accordance with Bree et al.'s definition of a crash $(25 \%$ drop in price) in [50, 51]. Hence, it seems that the approach by Bree et al. to compare the results of the fits when using the price (and not the log-price) is inconsistent.

One can also investigate the possibility that price changes may not be proportional to price. If this is the case, use of the real price is warranted according to the arguments put forward by Sornette and Johansen [1]. In practice, it is useful to try both fitting procedures with prices and log-prices and compare their relative merits. But one should be cautious because the fits using prices (and not log-prices) involve data values that may change over several orders of magnitude over the time window of interest. As a consequence, the standard least square fits is not suitable anymore. Instead, a normalized least square minimization is recommended so that each data point of the time series roughly contributes equally to the mean-square root diagnostic. This approach has been implemented recently in Ref. [80]. 


\section{Sloppiness of the JLS models and search algorithm}

Bree and Joseph [50] and Bree et al. [51] claim that the concept of sloppiness and its consequence should be considered in fitting the JLS model to empirical data with the LevenbergMarquart algorithm. They challenge the relevance of the obtained fits. We agree that sloppiness is real, but is not an issue with an appropriate methodology addressing it, and in view of the fact that the probabilistic predictions are in general consistent.

Specifically, a correct fitting procedure should include the combination of the LevenbergMarquart algorithm [81] and a preliminary taboo search [82] or other meta-heuristics such as the genetic algorithm and simulated annealing algorithm. This should occur together with the slaving of the linear parameters to the nonlinear ones in order to reduce the nonlinear searching space from 7 dimensions to 4 (and to 3 in the recently novel procedure of Filimonov and Sornette [83]). The taboo search is a very good algorithm that provides a robust preliminary systematic exploration of the space of solutions, which prevents the LevenbergMarquart algorithm later on being stuck in special regions of the space of solutions. Also in a standard fitting procedure, the many results that may be obtained from the taboo search (i.e. results associated with different parts of the searching space) should be kept.

Taking into account the two points mentioned above, the quality of the fits with the JLS model is furthermore checked by the visualization of the fits and the original data [17, 18, 34, 35, 80]. In contrast, it is obvious that fits using only the Levenberg-Marquart algorithm without a reasonable starting value and sufficient preliminary exploration of the space of solutions will produce spurious results, with the algorithm stuck in a local minimum at the boundary of the search space.

Bree and Joseph [50] provide a sensitivity analysis of the root mean square error (RMSE), in which one parameter is scanned while the others remain fixed. The problem is that, because of the nonlinearity of expression ([6), it is not obvious that the results of such a scan can be trusted. That is, if local minima in, say, $\omega$ are found while the other parameters are kept fixed, do the same minima appear when one or more of the other parameters are changed to different values? In other words, is the multi-dimensional parameter landscape around these minima smooth? The answer to this question is more important than showing the sensitivity of 2 dimensional subspaces, as in [50]. In practice, answering this question on the smoothness of the multi-dimensional parameter landscape is difficult. Filimonov and 
Sornette [83] have documented that the standard slaving of three linear parameters $(A, B$ and $C$ in expression ([6]) to the four remaining nonlinear parameters results in a corrugated fitness landscape that requires a meta-heuristic (such as the taboo search). This approach simultaneously changes all parameters in order to find acceptable minima as starting points for the Levenberg-Marquart algorithm. Yes, this approach does not guarantee finding the absolute minimum but it does provide an ensemble of acceptable local minima. This ensemble approach is more robust than searching in vain for a single global minimum.

\section{E. Performance of the recommended fitting method on synthetic data}

It is an essential building stone of any fitting procedure that it should be tested on synthetic data. Indeed, in any calibration exercise, one faces simultaneously two unknowns: (i) the performance, reliability and robustness of the calibration procedure and (ii) the time series under study from which one hopes to extract meaningful information. As a first step in the analysis, we explore how the fitting procedure performs when the underlying datagenerating process is known. Johansen et al. [2, 3] set the stage by developing comparative tests on synthetic time series generated by the GARCH model. We also attract the attention to the fact that one of the most extensive set of synthetic tests concerning the possible existence of spurious log-periodicity is found in reference [84]. Zhou and Sornette [85] presented a systematic study of the confidence levels for log-periodicity only, using synthetic time series with many different types of noises, including noises whose amplitudes are distributed according to power law distributions with different exponents and long-memory modeled by fractional Brownian noises with various Hurst exponents spanning the full range from anti-persistent $(0<H<1 / 2)$ to persistent $(1 / 2<H<1)$.

We now show that the current fitting methods estimate the parameters of the JLS model within a reasonable range of uncertainty. For this, a reference log-periodic power law (LPPL) time series of duration equal to 240 days is generated for a typical set of parameters, shown in Table I. This series corresponds to a value of the critical time $t_{c}$ equal to 300 (days). The choice of 240 days for the time window size is motivated by the typical length for the generation of bubbles found in various case studies in the literature. The choice of $\omega=10$ is in the range often found in empirical studies. We note that there are actually several ranges for $\omega$ corresponding to respectively the first, second and third harmonics of 


\begin{tabular}{|r|r|r|r|}
\hline & Reference & Mean (std) of Gaussian & Mean (std) of Student's t \\
\hline$t_{c}$ & 300 & $296.07(20.44)$ & $295.15(20.81)$ \\
\hline$m$ & 0.7 & $0.74(0.15)$ & $0.72(0.18)$ \\
\hline$\omega$ & 10 & $9.75(1.43)$ & $9.71(1.47)$ \\
\hline
\end{tabular}

TABLE I: The parameter values used to generate the synthetic data are shown in the second column "Reference". The mean and standard deviation values of the parameters obtained by fitting the JLS model to the synthetic LPPL time series decorated by the two types of noise discussed in the text are given in the last two columns. These numbers are estimated from 200 statistical realizations of the noise, and each realization is characterized by ten different best fits with the Levenberg-Marquart algorithm, leading to a total of 2000 estimated parameters. The other parameters used to generate the synthetic LPPL are $\phi=1, A=10, B=-0.1, C=0.02$.

the log-periodicity, as documented for instance in Refs. [20, 26, 55, 75, 79, 86].

The synthetic data is generated by combining the LPPL time series with noise. Two kinds of noise are considered: Gaussian noise and noise generated with a Student t distribution with four degrees of freedom (which exhibits a tail similar to that often reported in the literature for the distribution of financial returns). For both types of noise, the mean value is zero and the standard deviation is set to be $5 \%$ of the largest log-price among the 240 observations in the reference series. The standard deviation is chosen quite high in order to offer a stringent test of the efficiency of the current fitting method. Synthetic samples obtained with both types of noise along with the reference time series are shown in Fig. 2.

For each type of noise, 200 synthetic time series are generated. We fit each series with the JLS model (6) and keep the ten best fits for each one. Recall that our stochastic fitting method produces multiple 'good' fits instead of the 'best' fit, which, in practice, is difficult, if not impossible, to find. In the new procedure recently developed by Filimonov and Sornette [83], the 'best' fit can be found in most cases that are qualified to be in a bubble regime. However, we still use the standard heuristic procedure in the present paper, which predates that of Filimonov and Sornette [83]. The best ten selections result in 2000 sets of estimated parameters for each type of noise. The sampling distributions of $t_{c}, m$ and $\omega$ for the two types of noise are calculated by a non-parametric method (adaptive kernel technique). The results are shown is Fig. 3 . 
The mean and standard deviation of these parameters are shown in Table I alongside the original numbers used to generate the true LPPL function $\left(t_{c}=300, m=0.7, \omega=10\right)$ without noise. This test on synthetic data demonstrates that the fitting method combining the meta-heuristic Taboo search with the Levenberg-Marquart algorithm is satisfactory. We observe negligible biases, especially for the crucial critical time parameter $t_{c}$. The standard deviation for $t_{c}$ of about 20 days is three times smaller than the 60 days separating the last observation (day 240) of the time series and the true critical time occurring at the 300-th day, showing that the calibration of a time series exhibiting LPPL structure, even with very large statistical noise, can provide significant skills in forecasting the critical time $t_{c}$.

\section{PROBABILISTIC FORECAST}

From a practical risk management view point, one of the benefits obtained from the calibration of the JLS model to financial time series is the estimation of the most probable time of the end of the bubble $t_{c}$, which can take the form of a crash, but can also be a smooth transition to a new market regime.

As we mentioned before, a distribution of $t_{c}$ is obtained for a single bubble period, associated with the set of fitted time windows (see Section (IIB) and the recording of multiple locally optimal fits from the stochastic taboo search (see Section IIID). Recall that the output of the meta-heuristic is used as the initial guess required by the Levenberg-Marquart algorithm. As demonstrated in the previous subsection, the estimation of the distribution of the most probable time $t_{c}$ for the end of the bubble is generated by a reliable non-parametric method [87].

Bree et al. [51] make the interesting remark that the estimation of the probability density of $t_{c}$ might be improved by augmenting the analysis of the original time series with that of many replicas. These replicas of the initial time series can be obtained for instance by using a LPPL function obtained from the first calibration on the original time series and adding to it noise generated by an $\mathrm{AR}(1)$ process. This methodology provides a measure of robustness of the whole estimation exercise. The choice of an $\mathrm{AR}(1)$ process for the noise is supported by the evidence provided in Refs. [53, 88] that the residuals of the calibration of the JLS model to a bubble price time series can be reasonably described by an AR(1) process.

However this is only one among several possibilities. Another method, which we have 
implemented in our group for quite some time and now use systematically, is to generate bootstraps in which the residuals of the first calibration on the original time series are used to seed as many synthetic time series as needed, using reshuffled blocks of residuals of different durations. For instance, reshuffling residuals in blocks of 25 days ensures that the dependence structure between the residuals is identical in the synthetic time series as in the original one up to a month time scale. Note that this bootstrap method does not assume Gaussian residuals in contrast with the $\mathrm{AR}(1)$ noise generation model. It captures also arguably better the dependence structure of the genuine residuals than the linear correlation embedded in the $\mathrm{AR}(1)$ model.

\section{CONCLUSION}

We have discussed the present theoretical status and some calibration issues concerning the Johansen-Ledoit-Sornette (JLS) model of rational expectation bubbles with finite-time singular crash hazard rates. We have provided a guide to the advances that have punctuated the development of tests of the JLS model performed on a variety of financial markets during the last decade. We can say that the development of new versions and of methodological improvements have paralleled the occurrence of several major market crises, which have served as inspirations and catalyzers of the research. We believe that the field of financial bubble diagnostic [38-40, 89] is progressively maturing and we foresee in the near future that it could become operational to help decision makers alleviate the consequences of excess leverage leading to severe market dysfunctions.

Acknowledgement: Wei-Xing Zhou acknowledges financial support from the National Natural Science Foundation of China (11075054), and the Fundamental Research Funds for the Central Universities. This work was partially supported by ETH Research Grant ETH-31 10-3 "Testing the predictability of financial bubbles and of systemic instabilities". 


\section{Reference}

[1] A. Johansen, D. Sornette, Critical crashes, Risk 12 (1) (1999) 91-94.

[2] A. Johansen, D. Sornette, O. Ledoit, Predicting financial crashes using discrete scale invariance, Journal of Risk 1 (4) (1999) 5-32.

[3] A. Johansen, O. Ledoit, D. Sornette, Crashes as critical points, International Journal of Theoretical and Applied Finance 3 (2000) 219-255.

[4] D. Sornette, Why Stock Markets Crash (Critical Events in Complex Financial Systems), Princeton University Press, 2003.

[5] K. R. Sircar, G. Papanicolaou, General black-scholes models accounting for increased market volatility from hedging strategies, Applied Mathematical Finance 5 (1998) 45-82.

[6] J. B. De Long, A. Shleifer, L. Summers, R. Waldmann, Positive feedback investment strategies and destabilizing rational speculation, Journal of Finance XLV (1990) 379-395.

[7] F. Black, An equilibrium model of the crash, NBER Macroecomonics Annual (1988) 269-276.

[8] A. Kalife, Portfolio insurance strategies by a large player, http://ssrn.com/abstract=683705 (2004).

[9] P. Pasquariello, The microstructure of currency markets: An empirical model of intraday return and bid-ask spread behavior, EFMA 2001 Lugano Meetings; Stern School of Business Working Paper. Available at SSRN: http://ssrn.com/abstract $=252510$ or http://dx.doi.org/10.2139/ssrn.252510 (2010).

[10] J. J. Angel, L. Harris, C. S. Spatt, Equity trading in the 21st century, Marshall School of Business Working Paper No. FBE 09-10, Available at SSRN: http://ssrn.com/abstract=1584026 or http://dx.doi.org/10.2139/ssrn.1584026 (2010).

[11] S. Mithas, M. S. Krishnan, Human capital and institutional effects in the compensation of information technology professionals in the united states, Management Science 54 (3) (2008) $415-428$.

[12] M. Gisler, D. Sornette, R. Woodard, Innovation as a social bubble: The example of the human genome project, Research Policy 40 (2011) 1412-1425.

[13] E. X. N. Li, D. Livdan, L. Zhang, Anomalies, Review of Financial Studies 22 (11) (2009) 
4301-4334.

[14] I. Fender, The impact of corporate risk management on monetary policy transmission: Some empirical evidence, BIS Working Paper No. 95. Available at SSRN: http://ssrn.com/abstract=849065 or http://dx.doi.org/10.2139/ssrn.849065 (2000).

[15] J. Atik, Basel ii: A post-crisis post-mortem, Transnational Law \& Contemporary Problems 19 (2011) 731.

[16] L. Allen, S. Chakraborty, W. Watanabe, Foreign direct investment and regulatory remedies for banking crises: Lessons from japan, Journal of International Business Studies 42 (7) (2011) 875-893.

[17] D. Sornette, R. Woodard, W.-X. Zhou, The 2006-2008 oil bubble: Evidence of speculation and prediction, Physica A 388 (2009) 1571-1576.

[18] Z.-Q. Jiang, W.-X. Zhou, D. Sornette, R. Woodard, K. Bastiaensen, P. Cauwels, Bubble diagnosis and prediction of the 2005-2007 and 2008-2009 Chinese stock market bubbles, Journal of Economic Behavior and Organization 74 (2010) 149-162.

[19] W.-X. Zhou, D. Sornette, Analysis of the real estate market in Las Vegas: Bubble, seasonal patterns, and prediction of the CSW indexes, Physica A 387 (2008) 243-260.

[20] W.-X. Zhou, D. Sornette, 2000-2003 real estate bubble in the UK but not in the USA, Physica A 329 (2003) 249-263.

[21] W.-X. Zhou, D. Sornette, Is there a real estate bubble in the US?, Physica A 361 (2006) $297-308$.

[22] J. Fry, Statistical modelling of financial crashes, Ph.D. thesis, University of Sheffield (2007).

[23] J. M. Fry, Bubbles and crashes in finance: A phase transition from random to deterministic behaviour in prices, Journal of Applied Research in Finance 2 (2010) 131-137.

[24] J. M. Fry, Modelling bubbles and crashes in economics: With an application to english house prices, Unpublished.

[25] A. Johansen, D. Sornette, Financial "anti-bubbles": log-periodicity in gold and Nikkei collapses, International Journal of Modern Physics C 10 (4) (1999) 563-575.

[26] D. Sornette, W.-X. Zhou, The US 2000-2002 market descent: How much longer and deeper?, Quantitative Finance 2 (6) (2002) 468-481.

[27] N. Vandewalle, M. Ausloos, P. Boveroux, A. Minguet, Visualizing the log-periodic pattern before crashes, European Physics Journal B 9 (1999) 355-359. 
[28] A. Clark, Evidence of log-periodicity in corporate bond spreads, Physica A 338 (2004) 585-595.

[29] P. Gnaciński, D. Makowiec, Another type of log-periodic oscillations on Polish stock market, Physica A 344 (2004) 322-325.

[30] M. Bartolozzi, S. Drożdż, D. Leinweber, J. Speth, A. Thomas, Self-similar log-periodic structures in Western stock markets from 2000, International Journal of Modern Physics C 16 (2005) 1347-1361.

[31] J. M. Fry, Exogenous and endogenous market crashes as phase transitions in complex financial systems, Eur. Phys. J. B 85 (2012) 405.

[32] R. Matsushita, S. da Silva, A. Figueiredo, I. Gleria, Log-periodic crashes revisited, Physica A 364 (2006) 331-335.

[33] S. Drożdż, J. Kwapień, P. Oświecimka, J. Speth, Current log-periodic view on future world market development, Acta Physica Polonica A 114 (2008) 539-546.

[34] W.-X. Zhou, D. Sornette, A case study of speculative financial bubbles in the South African stock market 2003-2006, Physica A 388 (2009) 869-880.

[35] W. Yan, R. Woodard, D. Sornette, Leverage bubble, Physica A 391 (2012) 180-186.

[36] A. Johansen, D. Sornette, Bubbles and anti-bubbles in Latin-American, Asian and western stock markets: an empirical study, International Journal of Theoretical and Applied Finance 4 (2001) 853-920.

[37] J. M. Fry, O. Masood, Modelling over-pricing and speculative bubbles: An application to emerging stock markets, Unpublished.

[38] D. Sornette, R. Woodard, M. Fedorovsky, S. Reimann, H. Woodard, W.-X. Zhou, The financial bubble experiment: Advanced diagnostics and forecasts of bubble terminations (the financial crisis observatory), http://arxiv.org/abs/0911.0454 (2010).

[39] D. Sornette, R. Woodard, M. Fedorovsky, S. Reimann, H. Woodard, W.-X. Zhou, The financial bubble experiment: Advanced diagnostics and forecasts of bubble terminations volume II (master document), http://arxiv.org/abs/1005.5675 (2010).

[40] R. Woodard, D. Sornette, M. Fedorovsky, The financial bubble experiment: Advanced diagnostics and forecasts of bubble terminations volume III (beginning of experiment + post-mortem analysis), http://arxiv.org/abs/1011.2882 (2011).

[41] Y. Malevergne, D. Sornette, Extreme Financial Risks (From Dependence to Risk Management), Springer, Heidelberg, 2006. 
[42] W.-X. Zhou, D. Sornette, Evidence of a worldwide stock market log-periodic anti-bubble since mid-2000, Physica A 330 (2003) 543-583.

[43] B. Podobnik, D. Wang, D. Horvatic, I. Grosse, H. E. Stanley, Time-lag cross-correlations in collective phenomena, Europhysics Letters 90 (6) (2010) 68001, doi:10.1209/0295$5075 / 90 / 68001$.

[44] D. Wang, B. Podobnik, D. Horvatic, H. E. Stanley, Quantifying and modeling long-range cross-correlations in multiple time series with applications to world stock indices, Phys. Rev. E 83 (2011) 046121.

[45] J. Feigenbaum, A statistical analysis of log-periodic precursors to financial crashes, Quantitative Finance 1 (2001) 346-360.

[46] G. Chang, J. Feigenbaum, A bayesian analysis of log-periodic precursors to financial crashes, Quantitative Finance 6 (2008) 15-36.

[47] G. Chang, J. Feigenbaum, Detecting log-periodicity in a regime-switching model of stock returns, Quantitative Finance 8 (2008) 723-738.

[48] H.-C. G. van Bothmer, C. Meister, Predicting critical crashes? A new restriction for the free variables, Physica A 320 (2003) 539-547.

[49] P. Geraskin, D. Fantazzini, Everything you always wanted to know about log-periodic power laws for bubble modeling but were afraid to ask, European Journal of Finance 19 (5) (2013) $366-391$.

[50] D. Bree, N. L. Joseph, Fitting the log periodic power law to financial crashes: a critical analysis, http://arxiv.org/abs/1002.1010v2 (2013).

[51] D. Bree, D. Challet, P. P. Peirano, Prediction accuracy and sloppiness of log-periodic functions, Quantitative Finance 13 (2) (2013) 275-280.

[52] O. Blanchard, M. Watson, Bubbles, rational expectations and speculative markets, in Crisis in the Economic and Financial Structure, ed. by P. Wachtel, Lexington, MA. (1982) 295-315.

[53] L. Lin, R. Ren, D. Sornette, A consistent model of 'explorative' financial bubbles with meanreversing residuals, http://arxiv.org/abs/0905.0128 (2009).

[54] D. Sornette, A. Johansen, Large financial crashes, Physica A 245 (1997) 411-422.

[55] W.-X. Zhou, D. Sornette, R. Hill, R. Dunbar, Discrete hierarchical organization of social group sizes, Proceedings of the Royal Society B 272 (2005) 439-444.

[56] K. Ide, D. Sornette, Oscillatory finite-time singularities in finance, population and rupture, 
Physica A 307 (2002) 63-106.

[57] L. Lin, D. Sornette, Diagnostics of rational expectation financial bubbles with stochastic mean-reverting termination times, The European Journal of Finance 19 (5) (2013) 344-365.

[58] D. Sornette, W.-X. Zhou, Predictability of large future changes in major financial indices, International Journal of Forecasting 22 (2006) 153-168.

[59] W. Yan, R. Woodard, D. Sornette, Diagnosis and prediction of rebounds in financial markets, Physica A 391 (2012) 1361-1380.

[60] W. Yan, R. Woodard, D. Sornette, Diagnosis and prediction of tipping points in financial markets: Crashes and rebounds, Physics Procedia 3 (2010) 1641-1657.

[61] W. Yan, R. Rebib, R. Woodard, D. Sornette, Detection of crashes and rebounds in major equity markets, International Journal of Portfolio Analysis and Management 1 (1) (2012) $59-79$.

[62] D. Sornette, A. Johansen, Significance of log-periodic precursors to financial crashes, Quantitative Finance 1 (4) (2001) 452-471.

[63] J. V. Andersen, D. Sornette, Fearless versus fearful speculative financial bubbles, Physica A 337 (1-2) (2004) 565-585.

[64] J. Galbraith, The great crash, 1929, Mariner Books, 1997.

[65] C. Kindleberger, Manias, Panics and Crashes: A History of Financial Crises, 4th Edition, Wiley, 2000.

[66] D. Sornette, Critical market crashes, Physics Reports 378 (2003) 1-98.

[67] R. Gurkaynak, Econometric tests of asset price bubbles: Taking stock, Journal of Economic Surveys 22 (1) (2008) 166-186.

[68] T. Lux, D. Sornette, On rational bubbles and fat tails, Journal of Money, Credit and Banking 34 (3) (2002) 589-610.

[69] A. Johansen, D. Sornette, Shocks, crashes and bubbles in financial markets, Brussels Economic Review (Cahiers economiques de Bruxelles) 53(2) (2010) 201-253.

[70] A. Johansen, D. Sornette, Finite-time singularity in the dynamics of the world population and economic indices, Physica A 294 (3-4) (2001) 465-502.

[71] D. Johansen, A.and Sornette, Evaluation of the quantitative prediction of a trend reversal on the Japanese stock market in 1999, International Journal of Modern Physics C 11 (2) (2000) 359-364. 
[72] W.-X. Zhou, D. Sornette, Testing the stability of the 2000-2003 US stock market "antibubble", Physica A 348 (2005) 428-452.

[73] S. Gluzman, D. Sornette, Log-periodic route to fractal functions, Physical Review E 65 (2002) 036142 .

[74] W.-X. Zhou, D. Sornette, Renormalization group analysis of the 2000-2002 anti-bubble in the US S\&P 500 index: Explanation of the hierarchy of 5 crashes and prediction, Physica A 330 (2003) 584-604.

[75] W.-X. Zhou, D. Sornette, Evidence of intermittent cascades from discrete hierarchical dissipation in turbulence, Physica D 165 (2002) 94-125.

[76] D. Sornette, W.-X. Zhou, Evidence of fueling of the 2000 new economy bubble by foreign capital inflow: Implications for the future of the US economy and its stock market, Physica A $332(6)(2004) 412-440$.

[77] W.-X. Zhou, D. Sornette, Fundamental factors versus herding in the 2000-2005 US stock market and prediction, Physica A 360 (2009) 459-483.

[78] W. Yan, R. Woodard, D. Sornette, Role of diversification risk in financial bubbles, Journal of Investment Strategies 1 (4) (2012) 63-83.

[79] W.-X. Zhou, D. Sornette, Non-parametric analyses of log-periodic precursors to financial crashes, International Journal of Modern Physics C 14 (8) (2003) 1107-1126.

[80] W. Yan, R. Woodard, D. Sornette, Inferring fundamental value and crash nonlinearity from bubble calibration, Quantitative Finance DOI:10.1080/14697688.2011.606824 (2012) (http://arxiv.org/abs/1011.5343).

[81] K. Levenberg, A method for the solution of certain non-linear problems in least squares, Quarterly Journal of Applied Mathematics II 2 (1944) 164-168.

[82] D. Cvijović, J. Klinowski, Taboo search: An approach to the multiple minima problem, Science 267 (5188) (1995) 664-666.

[83] V. Filimonov, D. Sornette, A stable and robust calibration scheme of the log-periodic power law model, Physica A 392 (17) (2013) 3698-3707.

[84] Y. Huang, A. Johansen, M. W. Lee, H. Saleur, D. Sornette, Artifactual log-periodicity in finite-size data: Relevance for earthquake aftershocks, Journal of Geophysical Research 105 (2000) 25451-25471.

[85] W.-X. Zhou, D. Sornette, Statistical significance of periodicity and log-periodicity with heavy- 
tailed correlated noise, International Journal of Modern Physics C 13(2) (2002) 137-170.

[86] W.-X. Zhou, D. Sornette, Antibubble and prediction of china's stock market and real-estate, Physica A 337 (1-2) (2004) 243-268.

[87] Q. Li, J. S. Racine, Nonparametric Econometrics: Theory and Practice, Princeton University Press, 2006.

[88] L. Gazola, C. Fernades, A. Pizzinga, R. Riera, The log-periodic-AR(1)-GARCH(1,1) model of financial crashes, European Physics Journal B 61 (2008) 355-362.

[89] D. Sornette, R. Woodard, Financial bubbles, real estate bubbles, derivative bubbles, and the financial and economic crisis, in: M. Takayasu, T. Watanabe, H. Takayasu (Eds.), New Approaches to the Analysis of Large-Scale Business and Economic Data, Proceedings of APFA7 (Applications of Physics in Financial Analysis), Springer, 2010, http://arxiv.org/abs/0905.0220. 


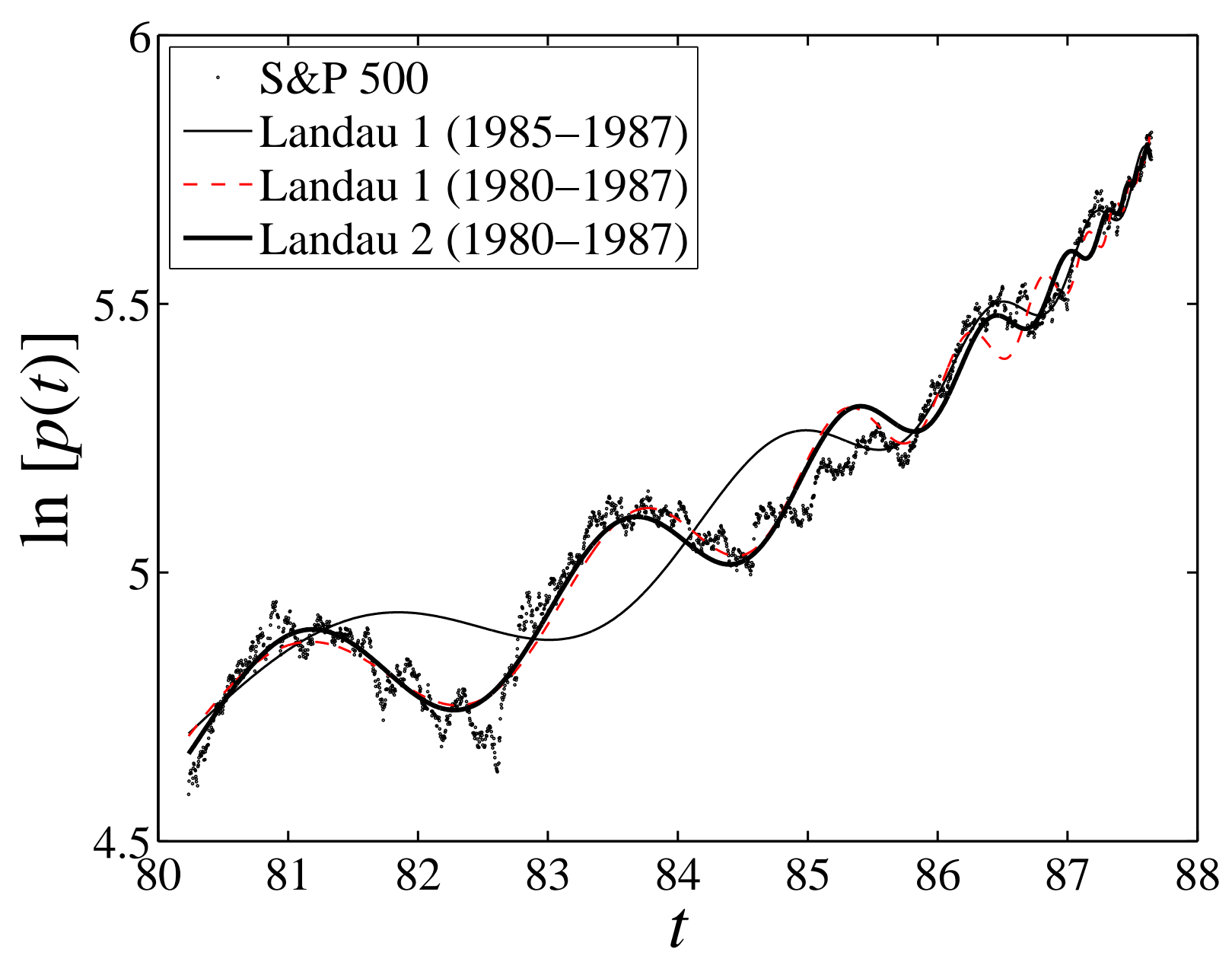

FIG. 1: Time dependence of the logarithm of the New York stock exchange index S\&P 500 from January 1980 to September 1987 and the best fits by the first and the second order LPPL Landau models. The crash of October 14, 1987 corresponds to 1987.78 decimal years. The thin continuous line represents the best fit with the first-order LPPL Landau model in the interval from July 1985 to the end of September 1987 and is shown on the whole time span since January 1980. The thin dashed line represents the best fit with the first-order LPPL Landau model in the interval from from January 1980 to September 1987. The thick line is the fit by the second-order LPPL Landau model in the interval from January 1980 to September 1987. (Reproduced from [54]) and extended). 

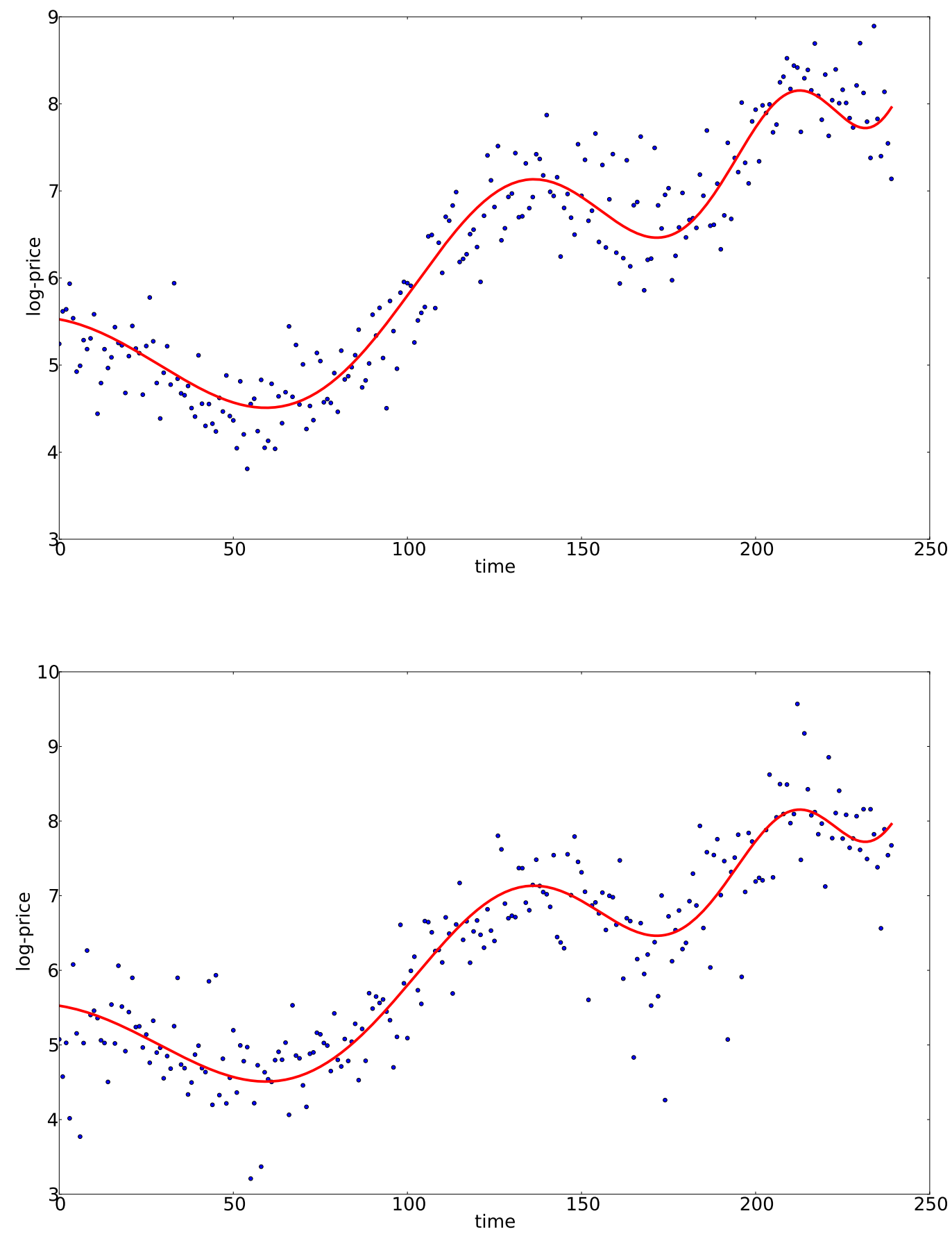

FIG. 2: Synthetic data examples with noise characterized by zero mean and large standard deviation ( $5 \%$ of the largest log-price among 240 reference points). Upper panel: the synthetic data with Gaussian noise. Lower panel: the synthetic data with noise generated with a Student t distribution with four degrees of freedom. The red solid line shows the reference LPPL time series. 

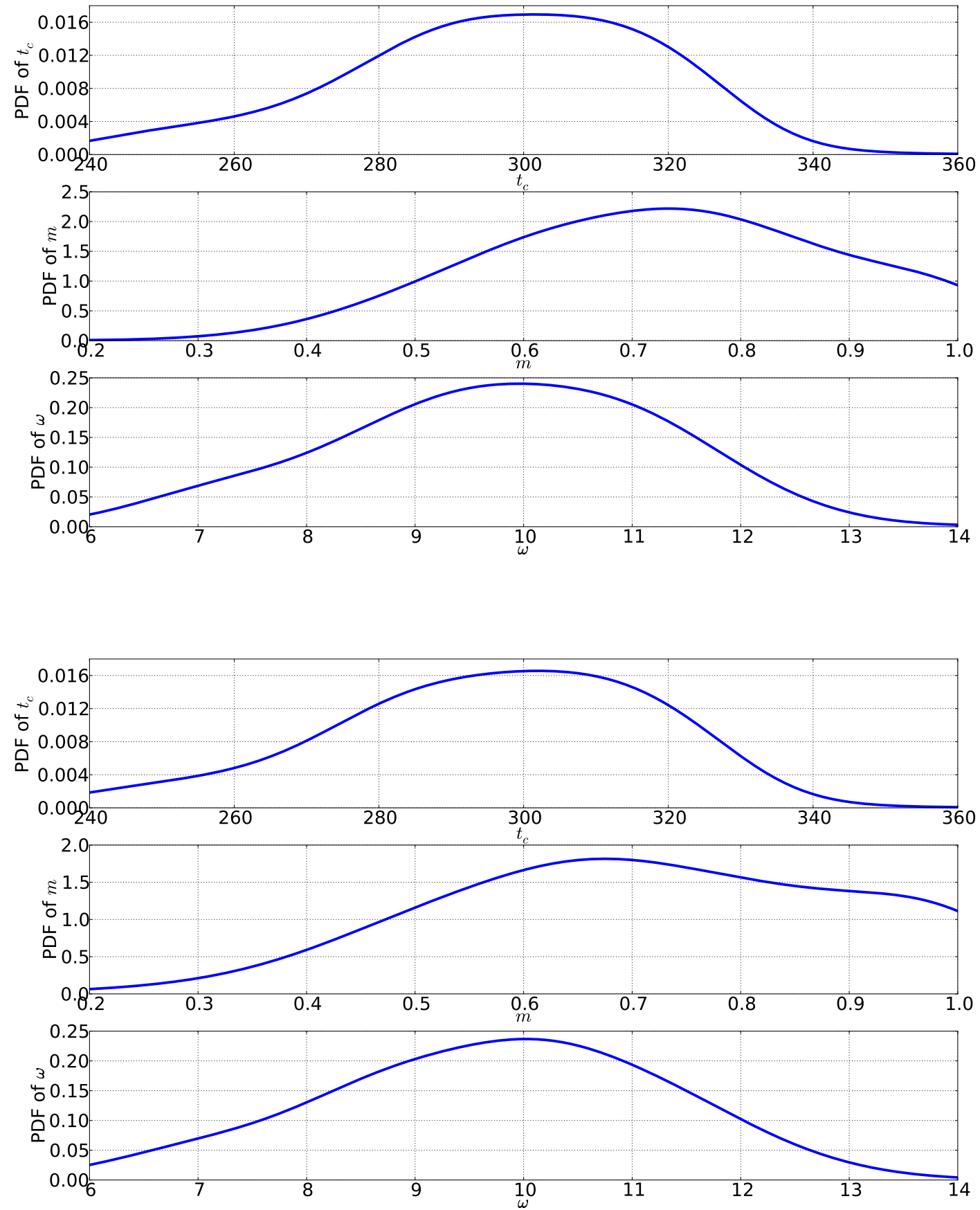

FIG. 3: The sampling distributions of $t_{c}, m$ and $\omega$ obtained by a non-parametric kernel method applied to the parameter values determined by analyzing 200 synthetic time series, each of which being characterized by its ten best fits with the Levenberg-Marquart algorithm, leading to a total of 2000 estimated parameters. Upper panel: the synthetic data with Gaussian noise. Lower panel: the synthetic data with noise generated with a Student $\mathrm{t}$ distribution with four degrees of freedom. 